\title{
Innovations in Retinal Laser Technology
}

\author{
Xinyi Xie ${ }^{1,2}$, Qinghuai Liu², Yannis M. Paulus ${ }^{1,3^{*}}$ \\ ${ }^{1}$ Department of Biomedical Engineering, University of Michigan, Ann Arbor, MI, USA \\ ${ }^{2}$ Department of Ophthalmology, The First Affiliated Hospital of Nanjing Medical University, Nanjing, China \\ ${ }^{3}$ Department of Ophthalmology and Visual Sciences, University of Michigan, Ann Arbor, MI, USA \\ Email: *ypaulus@med.umich.edu
}

How to cite this paper: Xie, X.Y., Liu, Q.H. and Paulus, Y.M. (2018) Innovations in Retinal Laser Technology. Optics and Photonics Journal, 8, 173-186. https://doi.org/10.4236/opj.2018.86016

Received: April 17, 2018

Accepted: June 18, 2018

Published: June 21, 2018

Copyright $\odot 2018$ by authors and Scientific Research Publishing Inc. This work is licensed under the Creative Commons Attribution International License (CC BY 4.0).

http://creativecommons.org/licenses/by/4.0/

\begin{abstract}
Retinal laser photocoagulation is a proven, effective treatment for various retinal disorders, including diabetic retinopathy, retinal vein occlusions, and sickle cell retinopathy. To minimize iatrogenic retinal damage while maintaining therapeutic effects, retinal laser technology has evolved significantly since its introduction in ophthalmology more than half a century ago. These innovations have included both optimizations of laser parameters in addition to the development of novel laser delivery systems. This review summarizes recent innovations in retinal laser technology, including subthreshold micropulse laser, selective retinal therapy and nanosecond laser, innovative modes of laser delivery including pattern scanning laser, endpoint management, navigated laser, and newly described photo-mediated ultrasound therapy.
\end{abstract}

\section{Keywords}

Retinal Laser Technology, Pattern Scan Laser, Navigated Laser, Subthreshold Micropulse Laser, Selective Retinal Therapy, Photo-Mediated Ultrasound Therapy, PASCAL

\section{Introduction}

Lasers are designed to produce a monochromatic, highly directional light beam with photons in phase and at the same frequency. These properties of lasers allow them to be a critical therapeutic tool in many fields of medicine, particularly in retinal diseases (Figure 1). Lasers have been used to treat various retinal conditions, including proliferative diabetic retinopathy, diabetic macular edema, central serous chorioretinopathy, central and branch retinal vein occlusions, sickle cell retinopathy, and age-related macular degeneration, since it was first described over 55 years ago [1]. Moreover, retinal laser photocoagulation has been the gold-standard of care for some of these retinal diseases for almost half a 


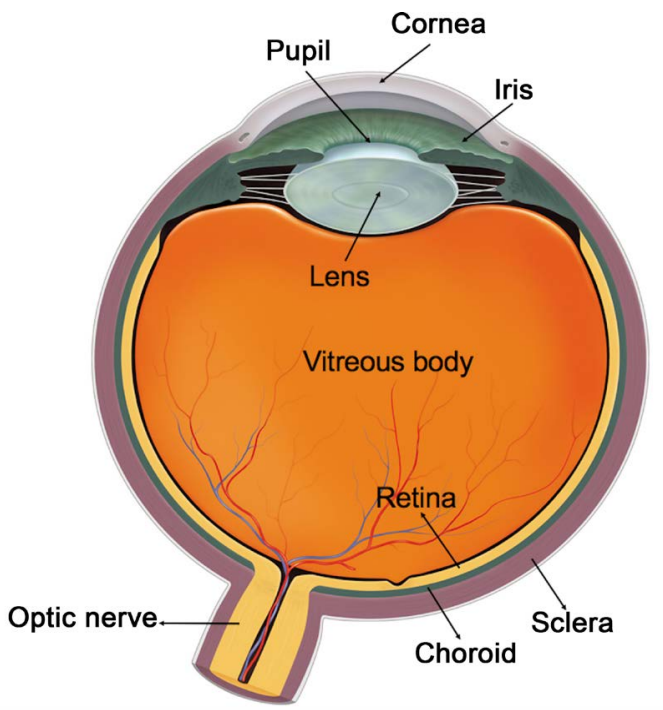

Figure 1. Schematic diagram of human eye.

century.

The precise biological mechanism by which laser therapy leads to improvement of retinal diseases remains an area of active investigation. One hypothesis is that photocoagulation destroys the most metabolically active cells, the photoreceptors, to relieve the imbalance between supply and demand of oxygen to decrease retinal hypoxia and subsequently causes down-regulation of angiogenic factors [2] [3]. Conventional retinal photocoagulation is typically performed with a $514-\mathrm{nm}$ or $532-\mathrm{nm}$ continuous wave $(\mathrm{cw})$ laser using pulse durations from 100 to 200 milliseconds (ms), spot sizes from 60 to 500 micrometers, and powers from 100 to 750 milliwatts $(\mathrm{mW})$. It produces detectable gray-white lesions in the retina as a desired therapeutic endpoint of treatment due to the thermal coagulation necrosis. These thermal effects are not only valuable for treating retinal diseases but also carrying significant side effects, such as permanent retinal scarring and decreased peripheral, color, and night vision [4].

Over the last couple decades, innovative laser modalities have significantly evolved, with refined laser parameters including laser wavelength, pulse duration and laser beam size, as well as novel laser delivery systems to minimize collateral tissue damage, improve therapeutic effects, and alleviate patients' discomfort [5] [6]. The purpose of this review is to summarize recent novel laser technologies for the treatment of retinal diseases.

\section{Pattern Scanning Laser}

An integrated semi-automatic pattern scanning laser photocoagulation system PASCAL $^{\circledR}$ (PAttern SCAnning Laser) photocoagulator was first described by Blumenkranz et al. in 2005 [7]. This system is designed to rapidly deliver laser pulses of 10 - 30 millisecond in a predetermined pattern array which allows for multiple spots of photocoagulation to be rapidly scanned with a single foot pedal application. PASCAL ${ }^{\circledR}$ consists of laser source such as a 532-nm Nd-YAG laser 
or a 514-nm argon laser, a delivery device such as a slit-lamp, and a control panel to select different laser parameters. The scanning of the laser is achieved by mirrors mounted on a two-axis galvanometric scanner.

PASCAL ${ }^{\circledR}$ is characterized by its scanning pattern and shorter pulse durations compared with conventional laser photocoagulation. The scanning pattern can be described as arrays of laser burns varying from 4 to 56 burns in different shapes and angles. The main benefit of the scanning pattern is that it makes laser therapy less painful and tedious due to shortened total treatment time. Furthermore, pre-specified, adjustable patterns provide greater accuracy during treatment. With shorter pulse duration, less thermal damage is caused to the inner retina [8] [9]. Human histological analysis of PASCAL laser burns has demonstrated that damage is limited to the outer retina [10] (Figure 2). Meanwhile, shorter exposures also allow equidistant spacing of numerous spots applied in a pattern during the eye fixation time [11] (Figure 3).

So far, several clinical trials have demonstrated PASCAL ${ }^{\circledR}$ to be effective with increased safety compared with conventional laser therapy in treating proliferative diabetic retinopathy (PDR) and other retinal vascular diseases [12] [13]. While some studies have found it to be less effective than conventional laser,

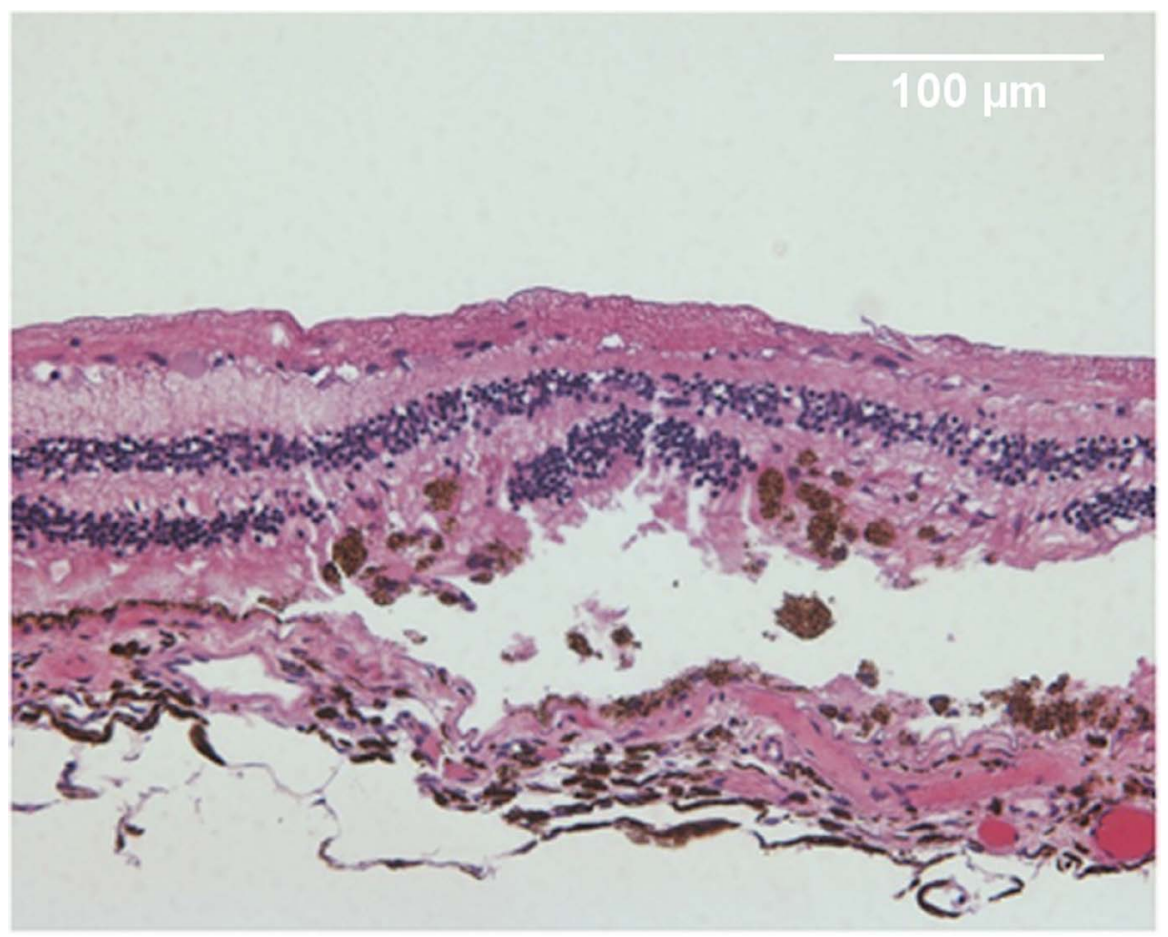

Figure 2. Hematoxylin and eosin $(\mathrm{H} \& \mathrm{E}) \times 10$ magnification section of PASCAL laser shows regions of outer nuclear layer loss filled with an acellular matrix and migrating pigmented cells within the outer retinal layers. There is disorganization of the choriocapillaris layer and areas of retinal pigment epithelial (RPE) atrophy and hyperplasia adjacent to these regions. The inner nuclear layer, ganglion cell layer, and nerve fiber layer appear preserved. Reprinted from "Human Histopathology of PASCAL Laser Burns", by Paulus, Y.M., Kaur, K., Egbert, P.R., Blumenkranz, M.S., \& Moshfeghi, D.M. 2013, Eye, 27(8): 995-996. 


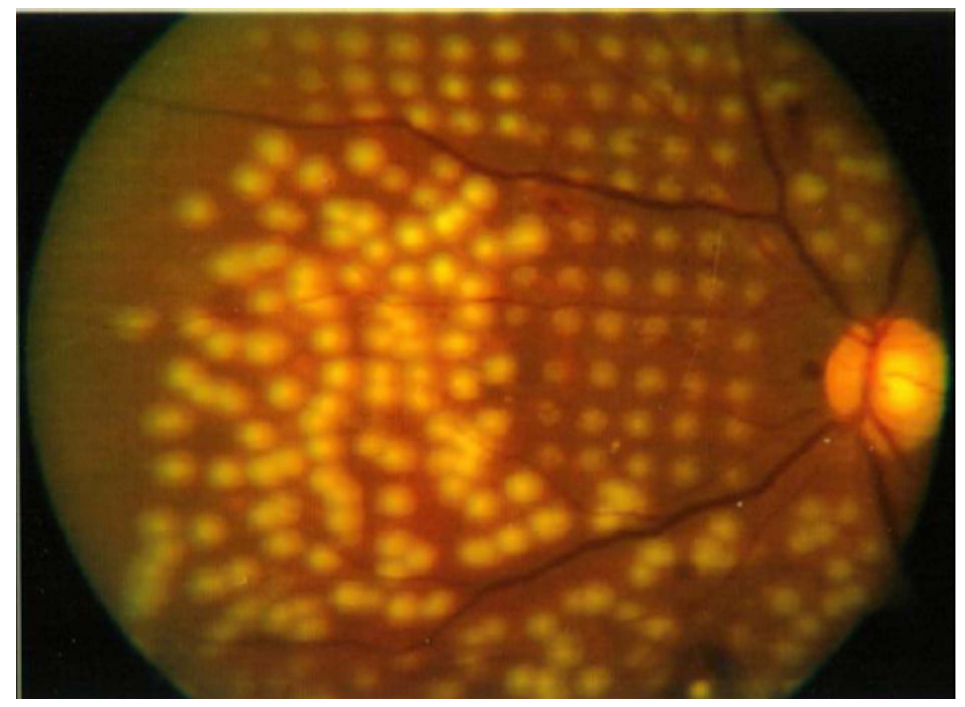

Figure 3. Fundus photograph comparing conventional laser (lower left laser spots in the image) with patterned scanning laser (upper right laser spots in the image, PASCAL, Topcon, Santa Clara, CA, USA) demonstrating more uniformly spaced, smaller, and less intense laser spots with PASCAL. Reprinted from "Short-pulse laser treatment: redefining retinal therapy”, by Paulus, Y. M., Palanker, D., \& Blumenkranz, M. S. 2010, Retinal Physician, 7(1), 54-56.

these studies have not accounted for the significant increase in the number of laser spots that are required to achieve an equivalent treatment area with shorter pulse duration laser burns [14] [15] [16]. PASCAL ${ }^{\circledR}$ is also an ideal laser system to treat patients with macular edema. Since patterns for macular photocoagulation can be predetermined as rings or arcs with a central foveal exclusion zone, PASCAL can help to make sure that no laser burns will be placed closer than a preset distance from the center of the foveal avascular zone [17] [18].

\section{Endpoint Management}

It is widely considered that the refined laser settings are favorable to reduce collateral tissue damage while retaining the therapeutic effects of laser. A model-based titration algorithm termed "Endpoint Management" (ЕpM) has been developed for quantifying laser dosimetry predictably to precisely optimize the laser power and pulse duration to achieve subvisible retinal laser therapy [19]. This titration algorithm combines an axisymmetric heat conduction model with the Arrhenius cellular damage integral to estimate heat-induced retinal lesions. To decrease the effects of individual variations, EpM begins with titrating laser power to a barely visible burn using pulse duration of 15 milliseconds or 20 milliseconds. After defining this energy as a $100 \%$ nominal energy level, other pulse energies can be selected as a percentage of that threshold. EpM is now commercially available in the 532-nm and 577-nm PASCAL ${ }^{\circledR}$ laser (Topcon Medical Laser Systems, Santa Clara, CA). This provides an approach to reproducible subvisible retinal laser therapy. Clinical trials have demonstrated that PASCAL ${ }^{\circledR}$ laser integrated with EpM software provides beneficial treatment effects for several retinal diseases such as chronic central serous chorioretinopathy (CSCR), macu- 
lar telangiectasia, diabetic macular edema (DME), and cystoid macular edema secondary to branch retinal vein occlusion (BRVO) without detectable retinal damage [20] [21].

\section{Targeted retinal photocoagulation}

It has been demonstrated that the mid-peripheral and peripheral retina are more prone to develop proliferative activities in PDR since capillary non-perfusion is more likely to happen in mid-peripheral and peripheral retina. To selectively treat areas of proliferative lesions in PDR, the strategy termed "targeted retinal photocoagulation" (TRP) has been developed [22] [23]. The target treatment makes retinal photocoagulation more accurate with reduced collateral damage to better-perfused areas of retina. Normally, a physician uses a slit-lamp or indirect ophthalmoscope to visualize the retina during retinal photocoagulation treatment. The emergence of the Optos scanning laser ophthalmoscopy (SLO) camera with ultra-wide field fluorescein angiography (Optos, Dunfermline, Scotland) enables the physician to visualize up to a $200^{\circ}$ field of view of the retina [22]. In pilot studies, patients with PDR were treated with 20-ms PASCAL ${ }^{\circledR}$ targeted retinal photocoagulation using 1500 laser burns. Optos angiography served to guide the PASCAL ${ }^{\circledR}$ photocoagulation lesion placement in areas of visible peripheral retinal ischemia. Also, the fluorescein angiography can be used to map laser burns on the retina [23]. The results showed that targeted PASCAL $^{\circledR}$ photocoagulation is a promising procedure with a favorable safety profile. In another clinical study, targeted $\mathrm{PASCAL}^{\circledR}$ retinal photocoagulation with high-density using 2500 burns was demonstrated to be safe without increased macular thickness or any other ocular adverse events during the short-term [24].

\section{Navigated Laser}

Another novel laser delivery system Navigated laser photocoagulation with retinal eye tracking (NAVILAS ${ }^{\circledR}$; OD-OS GmbH, Berlin) has been developed to offer more accurate and less painful laser photocoagulation using pre-planned, image-guided photocoagulation [25]. The navigated laser system combines a fundus imaging system with a laser treatment device and allows various imaging modalities, including infrared, color and fluorescein angiography, to be integrated with laser treatment of the retina. The NAVILAS ${ }^{\circledR}$ laser system fundamentally differs from other manual-technique laser devices since it is a fundus camera-based system instead of a slit lamp-based laser system. With the NAVILAS ${ }^{\circledR}$, the computer based imaging system can capture high-resolution images of a large retinal area at a rate of 25 images per second in real-time. The acquisition of retinal images before the treatment can help to create a detailed treatment plan through the touch-screen monitor. The pre-planned treatment is visible and overlaid on the live fundus images acquired during the treatment. Then the laser is delivered automatically to the target area of retina according to the plan, which allows for better guidance of laser delivery and high precision 
and reproducibility of theoretically less than $60-110 \mu \mathrm{m}$. Similar to conventional and pattern laser systems, navigated laser also includes both single-spot and preset patterns for retinal photocoagulation. For focal laser treatment, it utilizes a $50^{\circ}$ field-of-view for imaging. For panretinal laser treatment, the field-of-view can be increased to $85^{\circ}$ so that the peripheral retina can be visualized.

NAVILAS ${ }^{\circledR}$ has been successfully used in the treatment for several retinal vascular diseases, including diabetic retinopathy and CSCR [24] [26]. It has also been demonstrated that navigated laser produced more uniform laser burns with less pain and shorter treatment period compared with conventional and pattern scanning laser photocoagulation.

\section{Subthreshold Diode Micropulse Laser}

A novel technology termed subthreshold diode micropulse (SDM) photocoagulation was developed to treat retinal vascular disorders while minimizing collateral tissue damages using an 810-nm diode laser with micropulse duration [27]. SDM uses a continuous-wave laser which is divided into microsecond pulses to localize the thermal effects to the level of the retinal pigment epithelium (RPE), which lies between the neural retina and the choroidal layer and plays a vital role in the maintenance of the photoreceptor function. The term "subthreshold" refers to laser energy applied without visible intraretinal damage or scarring either during or after treatment. Laser burns produced by SDM do not appear on fluorescein angiography, which indicates the intact RPE tight junctions [28]. With micropulse duration, the increase in temperature of the neurosensory retina will not exceed the protein denaturation threshold when the minimum laser irradiance required for activation of the RPE is applied. Sublethally injured RPE is hypothesized to modify the expression of various cytokines that regulate angiogenesis and vascular leakage, which is believed to be responsible for the therapeutic effects of SDM [29].

Using a micropulse mode, laser is delivered with a train of short repetitive pulses separated by variable quiet intervals achieved by alteration of the duty cycle of laser. A duty cycle is the fraction of a period of time when the laser is "on". This "on" time is the duration of each micropulse (typically $100 \mu$ s to 300 $\mu \mathrm{s})$, and the "off" time (typically $1700 \mu$ s to $1900 \mu \mathrm{s}$ ) is the interval between micropulses, which allows for reduction of originated heat [30]. When a low duty cycle is used, commonly set at 5\% for SDM, the "on" time is short enough to allow the tissue return to baseline temperature between pulses, which also limits the time for thermal dissipation between each pulse thereby reducing collateral damage. To achieve the beneficial effects of laser, repeated pulses are added, allowing the tissue to return to baseline temperature between pulses.

The absence of collateral laser damage with micropulse laser may allow high-density therapy. Low-intensity/high-density micropulse laser is particular favorable for DME or ischemic retina with PDR. SDM was demonstrated as equal or superior to conventional laser photocoagulation for the treatment of 
DME and PDR without causing any adverse treatment effects or complications [31]. SDM have also been shown to avoid the risks of conventional laser with the ability to achieve similar clinical effects in patients with macular edema secondary to branch retinal vein occlusions and subretinal fluid from CSCR [32] [33].

\section{Selective Retinal Therapy (SRT)}

Conventional laser photocoagulation has limited use in many retinal diseases involving the macular such as age-related macular degeneration (AMD), DME, and CSCR due to collateral thermal damage which can cause severe loss of vision. These diseases are considered to be associated with the dysfunction of RPE. Selective retinal therapy (SRT) with microsecond pulses has been developed to selectively induce changes in the RPE so that the laser-induced thermal damage to surrounding tissue can be avoided, particularly to the neurosensory retina [34]. With microsecond pulses, produced heat is confined to the absorber site, predominantly the melanosomes within RPE cells. Formation of intracellular microbubbles around melanosomes results in destruction of RPE cells while avoiding damage to surrounding tissue. Subsequently, RPE cells proliferate and migrate into the lesion site to restore RPE continuity. The clinical potential of microsecond pulses in selectively destroying RPE cells was first demonstrated in rabbit eyes [35] [36]. It used 5-microsecond argon laser pulses at $514 \mathrm{~nm}$ and a repetition rate of $500 \mathrm{~Hz}$. Histologic analysis revealed that the damage in SRT lesions is primarily limited to the RPE while the surrounding retinal temperature remains at sublethal levels. Preliminary clinical trials performed with a Nd:YLF laser using a pulse duration of $1.7 \mu$ s have demonstrated no visual loss after the treatment, as confirmed by microperimetry [37].

An alternative mode of SRT has been developed which allows for the use of a currently available laser system using a rapidly scanning CW laser to produce microsecond laser dwell times to achieve the selective treatment of RPE cells [38]. Experiments with rabbits have demonstrated that line scanning mode of the PASCAL ${ }^{\circledR}$ laser can produce 15 -microsecond and 60 -microsecond retinal exposures which damages the RPE without a loss of overlying photoreceptors (Figure 4).

So far, the efficacy and safety of SRT have been established through several small clinical trials [39] [40]. In an international, multicenter clinical trial, SRT was performed on patients suffering from DME, CSCR, and drusen associated with AMD. One-year follow up after SRT, most of the patients underwent a reduction in symptoms [37].

\section{Retinal Rejuvenation Therapy}

Age-related macular degeneration (AMD) is now the leading cause of severe vision loss in adults over 50 years old worldwide. AMD is classified as either neovascular (Wet AMD) or non-neovascular (Dry AMD). Early AMD is characterized by three common pathologies including thickening of Bruch's membrane, 


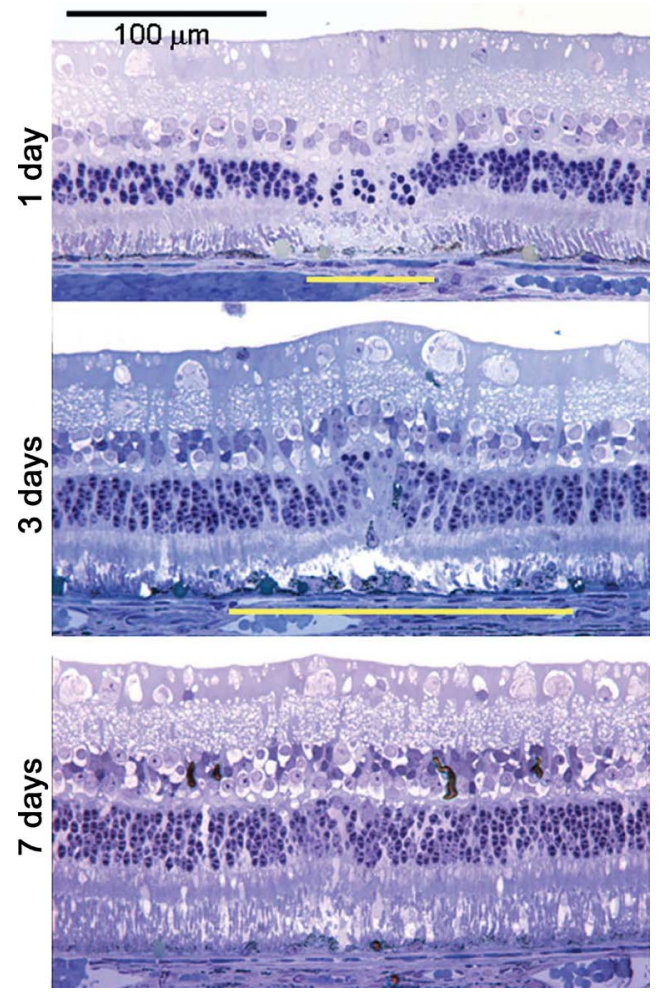

Figure 4. Healing of retinal lesions produced by line scanning laser with 15 microseconds dwell time. Histology of clinically invisible lesion at 1, 3, and 7 days after treatment. One day demonstrates RPE cell collapse and increased density of the outer segments. At 3 days, outer segments appear abnormal within a zone twice as wide than the initial lesion, with edema between the outer segments and RPE. By 1 week, the RPE defect and the damage to the outer segments have largely resolved. Reprinted from "Selective Retinal Therapy with Microsecond Exposures using a Continuous Line Scanning Laser", by Paulus, Y.M., Jain, A., Nomoto, H., Sramek, C., Gariano, R.F., Andersen, D., Schuele, G., Leung, L., Leng, T., \& Palanker, D. 2011, Retina, 31(2): 380-388.

deposits termed drusen under the RPE, and pigmentary changes in the macula. More advanced stages of AMD demonstrate either atrophy of RPE and retina and subretinal neovascularization, which are known as geographic atrophy (Dry AMD) and neovascular AMD respectively. The dysfunction and loss of RPE cells is regarded as the main pathological changes of AMD [41]. Although there are effective treatments available for patients with wet AMD such as anti-vascular endothelial growth factor (anti-VEGF) agents, there are currently no approved medical or surgical treatments available for patients with dry AMD, which accounts for the majority of AMD cases, although this is an area of active investigation with several active clinical trials.

An innovative, non-thermal, ultra-low energy, short-pulse (3 nanoseconds), 532-nm laser, the Retinal Rejuvenation Therapy laser (2RT ${ }^{\mathrm{TM}}$; Ellex Medical Lasers Ltd.), has been recently developed as a novel method to reduce the number of drusen and thus delay the progression of early AMD [42]. Compared with SDM and SRT, this technology uses even shorter pulse duration laser to confine the laser energy to the RPE and thus reduce collateral thermal damage [43]. 
During the treatment, the speckle beam profile of nanosecond laser targets individual RPE cells, which causes intracellular damage through microbubble growth and subsequent expansion around endogenous melanosomes in RPE cells. The nanosecond laser-induced RPE injury is intended to stimulate a biological regeneration and migration of RPE cells without collateral tissue damage. Several clinical trials have demonstrated the potential of $2 \mathrm{RT}^{\mathrm{TM}}$ to reverse pathologic changes in patients with early AMD, preventing neovascularization and progression to wet AMD [44] [45]. The 2RT laser, at the subthreshold setting, has also been shown to be as effective as conventional retinal photocoagulation in reducing macular edema secondary to diabetic retinopathy [46].

\section{Photo-Mediated Ultrasound Therapy (PUT)}

Numerous ocular diseases are associated with neovascularization. However, currently available anti-vascular treatments such as anti-VEGF agents and photodynamic therapy (PDT) have several drawbacks. Recently, our group developed a novel, selective, localized anti-vascular technique called photo-mediated ultrasound therapy (PUT) by applying synchronized nanosecond laser irradiation and ultrasound pulses. The high precision of PUT is closely related to its underlying mechanism which is photoacoustic cavitation, or more generally, the photospallation effect. When pulsed laser energy is absorbed by blood, photospallation may produce cavitation through strong, transient thermal-elastic stress. Moreover, in an object with spherical or cylindrical shape (e.g. a blood vessel), the laser-induced photoacoustic wave can converge into the center and achieve a significantly high acoustic pressure and produce cavitation, which is referred to as "cold bubbles" and has been observed in cells. The addition of a laser pulse to an existing ultrasound field can significantly improve the likelihood of localized inertial cavitation [47].

Taking advantage of the high optical absorption of hemoglobin, PUT can selectively target microvessels without causing unwanted damage to the surrounding tissue (Figure 5). In PUT, the required energy levels of both ultrasound and laser are significant lower than those used in previous therapies. Therefore, no significant temperature rise is induced and thermal damage can be effectively avoided. Moreover, PUT is noninvasive and agent-free unlike PDT.

Pre-clinical studies in rabbits has shown that PUT holds significant potential for the management retinal and choroidal vascular diseases in future, but further investigation and clinical trials are needed.

\section{Conclusions}

Retinal laser therapy has been playing a critical role in the care for numerous retinal diseases for over 50 years. Conventional laser photocoagulation has significant side effects. There have been significant modifications and novel laser modalities developed to achieve better outcomes while minimizing the side effects of conventional retinal laser therapy (Table 1). 


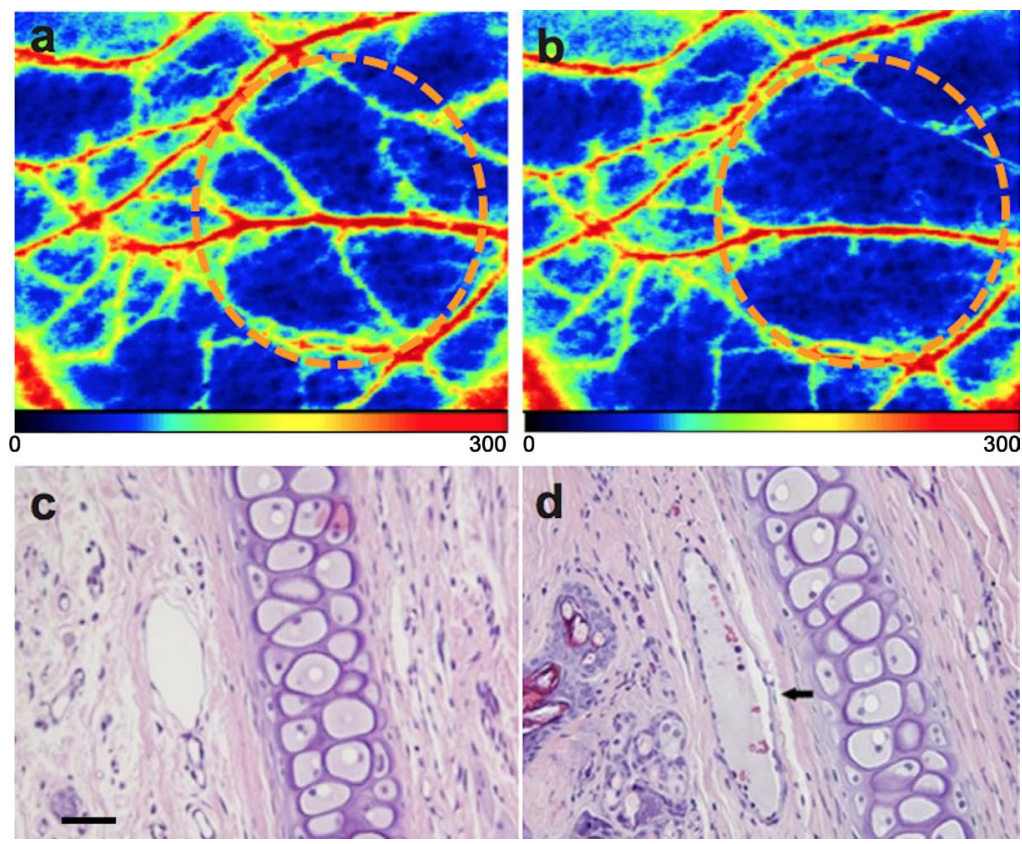

Figure 5. Effect of PUT on a rabbit ear model in vivo and histological photographs of rabbit ear tissues. ((a), (b)) Examples of the blood perfusion maps measured by a PeriCam PSI System before and after PUT on a rabbit ear. The perfusion map clearly demonstrated diminished blood flow in the treated microvessels after the treatment. Orange circled indicate treated areas. ((c), (d)) H \& E stain images of rabbit ear tissue without and with PUT treatment respectively. The formation of fibrin clot (black arrow) within the lumen in an auricular vein can be noticed, while the surrounding cells outside the blood vessel are not damaged. Scale bar: $50 \mu \mathrm{m}$. Reprinted from "High-precision, non-invasive anti-microvascular approach via concurrent ultrasound and laser irradiation", by $\mathrm{Hu}, \mathrm{Z}$., Zhang, H., Mordovanakis, A., Paulus, Y.M., Liu, Q., Wang, X., \& Yang, X. 2017, Scientific Reports, Jan 11; 7: 40243.

Table 1. Comparison of various novel retinal laser techniques.

\begin{tabular}{|c|c|c|c|c|c|}
\hline & PASCAL $^{\circledR}$ & NAVILAS $^{\circledR}$ & SDM & SRT & $2 \mathrm{RT}^{\circledR}$ \\
\hline Laser devices & $\begin{array}{c}\text { 532-nm } \\
\text { Nd-YAG/514-nm argon } \\
\text { laser }\end{array}$ & 577-nm Yellow laser & 810-nm diode laser & $\begin{array}{c}\text { 527-nm Nd-YLF/532-nm } \\
\text { Nd-YAG laser }\end{array}$ & 532-nm Nd-YAG laser \\
\hline Pulse duration & $10-1000 \mathrm{msec}$ & $10-1000 \mathrm{msec}$ & $100-300 \mu \mathrm{sec}$ & $1.7 \mu \mathrm{sec} / 15-60 \mathrm{msec}$ & $3 \mathrm{nsec}$ \\
\hline Indications & $\mathrm{PDR} / \mathrm{DME} / \mathrm{RVO}$ & PDR/DME/RVO & CSCR/DME & CSCR/DME & Early AMD \\
\hline Advantages & $\begin{array}{l}\text { Shortened treatment } \\
\text { time/Increased safety }\end{array}$ & $\begin{array}{l}\text { Eye-tracking/Better } \\
\text { accuracy }\end{array}$ & $\begin{array}{c}\text { Minimized collateral tissue } \\
\text { damage }\end{array}$ & $\begin{array}{c}\text { Selectively damage RPE } \\
\text { cells }\end{array}$ & $\begin{array}{c}\text { Confine laser energy to } \\
\text { RPE cells }\end{array}$ \\
\hline Limitations & $\begin{array}{l}\text { Uncontrolled eye } \\
\text { movements }\end{array}$ & $\begin{array}{c}\text { No stereoscopic } \\
\text { view/Unavailability } \\
\text { of ICG angiography }\end{array}$ & $\begin{array}{c}\text { Long treatment } \\
\text { time/Treatment protocols } \\
\text { not well-established }\end{array}$ & $\begin{array}{l}\text { Lack of non-invasive } \\
\text { methods to detect } \\
\text { treatment effects }\end{array}$ & $\begin{array}{l}\text { No visible reaction on } \\
\mathrm{RPE} / \mathrm{Impossible} \mathrm{to} \\
\text { determine the treated area }\end{array}$ \\
\hline
\end{tabular}

Pattern scanning laser photocoagulation $\left(\mathrm{PASCAL}^{\circledR}\right)$ was developed to rapidly apply numerous spots in a defined pattern to reduce treatment time, increase patient comfort, and improve the accuracy of treatment. Navigated laser has further improved the treatment accuracy using image-guidance. Micropulse and nanosecond pulse duration laser have shortened laser pulses to cause more loca- 
lized laser-induced effects and reduce unwanted thermal damage to surrounding tissues.

The development of these novel technologies demonstrates that lasers will continue to play a critical role in the treatment of retinal diseases. In the future, further investigations will also be made to evaluate the effect of combined therapy. Concurrent therapy of laser and pharmacologic agents has been shown to not be simply additive, but the laser and pharmacotherapies can affect the outcomes of one another. More recently, a newly described laser technology PUT combines laser irradiation with ultrasound bursts shows great potential for the management of neovascular diseases [48]. Continuing innovations in laser technology and improved understanding of laser-retinal interactions make us believe that laser therapy will continue to play a critical role in the treatment of retinal diseases for many years to come.

\section{References}

[1] Palanker, D.V., Blumenkranz, M.S. and Marmor, M.F. (2011) Fifty Years of Ophthalmic Laser Therapy. Archives of Ophthalmology, 129, 1613-1619.

https://doi.org/10.1001/archophthalmol.2011.293

[2] Palanker, D., Lavinsky, D., Blumenkranz, M.S. and Marcellino, G. (2011) The Impact of Pulse Duration and Burn Grade on Size of Retinal Photocoagulation Lesion: Implications for Pattern Density. Retina, 31, 1664-1669. https://doi.org/10.1097/IAE.0b013e3182115679

[3] Paulus, Y.M., Jain, A., Gariano, R.F., Stanzel, B.V., Marmor, M., Blumenkranz, M.S., et al. (2008) Healing of Retinal Photocoagulation Lesions. Investigative Ophthalmology \& Visual Science, 49, 5540-5545. https://doi.org/10.1167/iovs.08-1928

[4] Paulus, Y.M. (2015) New Frontiers in Selective Retinal Lasers. International Journal of Ophthalmic Research, 1, 1-4. https://doi.org/10.17554/j.issn.2409-5680.2015.01.8

[5] Sramek, C., Leung, L.S., Leng, T., Brown, J., Paulus, Y.M., Schuele, G., et al. (2011) Improving the Therapeutic Window of Retinal Photocoagulation by Spatial and Temporal Modulation of the Laser Beam. Journal of Biomedical Optics, 16, Article ID: 028004. https://doi.org/10.1117/1.3542045

[6] Sramek, C.K., Leung, L.S., Paulus, Y.M. and Palanker, D.V. (2012) Therapeutic Window of Retinal Photocoagulation with Green (532-nm) and Yellow (577-nm) Lasers. Ophthalmic Surgery, Lasers and Imaging Retina, 43, 341-347. https://doi.org/10.3928/15428877-20120426-05

[7] Blumenkranz, M.S., Yellachich, D., Andersen, D.E., Wiltberger, M.W., Mordaunt, D., Marcellino, G.R., et al. (2006) Semiautomated Patterned Scanning Laser for Retinal Photocoagulation. Retina, 26, 370-376. https://doi.org/10.1097/00006982-200603000-00024

[8] Jain, A., Blumenkranz, M.S., Paulus, Y., Wiltberger, M.W., Dan, E.A., Huie, P., et al. (2008) Effect of Pulse Duration on Size and Character of the Lesion in Retinal Photocoagulation. Archives of Ophthalmology, 126, 78-85.

https://doi.org/10.1001/archophthalmol.2007.29

[9] Sramek, C., Paulus, Y., Nomoto, H., Huie, P., Brown, J. and Palanker, D. (2009) Dynamics of Retinal Photocoagulation and Rupture. Journal of Biomedical Optics, 14, Article ID: 034007. https://doi.org/10.1117/1.3130282 
[10] Paulus, Y.M., Kaur, K., Egbert, P.R., Blumenkranz, M.S. and Moshfeghi, D.M. (2013) Human Histopathology of PASCAL Laser Burns. Eye, 27, 995-996. https://doi.org/10.1038/eye.2013.100

[11] Paulus, Y.M., Palanker, D. and Blumenkranz, M.S. (2010) Short-Pulse Laser Treatment: Redefining Retinal Therapy. Retinal Physician, 7, 54-59.

[12] Muqit, M.M., Marcellino, G.R., Henson, D.B., Young, L.B., Patton, N., Charles, S.J., et al. (2010) Single-Session vs Multiple-Session Pattern Scanning Laser Panretinal Photocoagulation in Proliferative Diabetic Retinopathy: The Manchester Pascal Study. Archives of Ophthalmology, 128, 525-533. https://doi.org/10.1001/archophthalmol.2010.60

[13] Muqit, M.M., Sanghvi, C., Mclauchlan, R., Delgado, C., Young, L.B., Charles, S.J., et al. (2012) Study of Clinical Applications and Safety for Pascal ${ }^{\circledR}$ Laser Photocoagulation in Retinal Vascular Disorders. Acta Ophthalmologica, 90, 155-161. https://doi.org/10.1111/j.1755-3768.2009.01854.x

[14] Chappelow, A.V., Tan, K., Waheed, N.K. and Kaiser, P.K. (2012) Panretinal Photocoagulation for Proliferative Diabetic Retinopathy: Pattern Scan Laser versus Argon Laser. American Journal of Ophthalmology, 153, 164-170. https://doi.org/10.1016/j.ajo.2011.05.035

[15] Bressler, S.B., Beaulieu, W.T., Glassman, A.R., Gross, J.G., Jampol, L.M., Melia, M., et al. (2017) Factors Associated with Worsening Proliferative Diabetic Retinopathy in Eyes Treated with Panretinal Photocoagulation or Ranibizumab. Ophthalmology, 124, 431-439. https://doi.org/10.1016/j.ophtha.2016.12.005

[16] Thomas, M., Rao, R.C., Johnson, M.W. and Paulus, Y.M. (2017) Re: Bressler et al.: Factors Associated with Worsening Proliferative Diabetic Retinopathy in a Eyes Treated with Panretinal Photocoagulation or Ranibizumab. (Ophthalmology. 2017; 124: 431-439). Ophthalmology, 124, e87-e88. https://doi.org/10.1016/j.ophtha.2017.06.025

[17] Modi, D., Chiranand, P. and Akduman, L. (2009) Efficacy of Patterned Scan Laser in Treatment of Macular Edema and Retinal Neovascularization. Clinical Ophthalmology, 3, 465-470. https://doi.org/10.2147/OPTH.S6486

[18] Muqit, M.M., Gray, J.C., Marcellino, G.R., Henson, D.B., Young, L.B., Patton, N., et al. (2010) Barely Visible 10-Millisecond Pascal Laser Photocoagulation for Diabetic Macular Edema: Observations of Clinical Effect and Burn Localization. American Journal of Ophthalmology, 149, 979-986.e2.

https://doi.org/10.1016/j.ajo.2010.01.032

[19] Lavinsky, D., Sramek, C., Wang, J., Huie, P., Dalal, R., Mandel, Y., et al. (2014) Subvisible Retinal Laser Therapy: Titration Algorithm and Tissue Response. Retina, 34, 87-97. https://doi.org/10.1097/IAE.0b013e3182993edc

[20] Lavinsky, D., Wang, J., Huie, P., Dalal, R., Lee, S.J., Lee, D.Y., et al. (2016) Nondamaging Retinal Laser Therapy: Rationale and Applications to the Macula. Investigative Ophthalmology \& Visual Science, 57, 2488-2500. https://doi.org/10.1167/iovs.15-18981

[21] Palanker, D. (2014) Non-Damaging Laser Therapy of the Macula: Titration Algorithm and Tissue Response. Proceedings of SPIE-The International Society for Optical Engineering, 218, 2458-2462.

[22] Muqit, M.M., Marcellino, G.R., Henson, D.B., Young, L.B., Patton, N., Charles, S.J., et al. (2013) Optos-Guided Pattern Scan Laser (Pascal)-Targeted Retinal Photocoagulation in Proliferative Diabetic Retinopathy. Acta Ophthalmologica, 91, 251-258. https://doi.org/10.1111/j.1755-3768.2011.02307.x 
[23] Reddy, S., Hu, A. and Schwartz, S.D. (2009) Ultra Wide Field Fluorescein Angiography Guided Targeted Retinal Photocoagulation (TRP). Seminars in Ophthalmology, 24, 9-14. https://doi.org/10.1080/08820530802519899

[24] Kozak, I., Kim, J.S., Oster, S.F., Chhablani, J. and Freeman, W.R. (2012) Focal Navigated Laser Photocoagulation in Retinovascular Disease: Clinical Results in Initial Case Series. Retina, 32, 930-935. https://doi.org/10.1097/IAE.0b013e318227ab5b

[25] Kernt, M., Cheuteu, R., Vounotrypidis, E., Haritoglou, C., Kampik, A., Ulbig, M.W., et al. (2011) Focal and Panretinal Photocoagulation with a Navigated Laser $\left(\right.$ NAVILAS $^{\circledR}$ ). Acta Ophthalmologica, 89, e662-e664. https://doi.org/10.1111/j.1755-3768.2010.02017.x

[26] Kozak, I., Oster, S.F., Cortes, M.A., Dowell, D., Hartmann, K., Kim, J.S., et al. (2011) Clinical Evaluation and Treatment Accuracy in Diabetic Macular Edema Using Navigated Laser Photocoagulator NAVILAS. Ophthalmology, 118, 1119-1124. https://doi.org/10.1016/j.ophtha.2010.10.007

[27] Sivaprasad, S., Elagouz, M., Mchugh, D., Shona, O. and Dorin, G. (2010) Micropulsed Diode Laser Therapy: Evolution and Clinical Applications. Survey of Ophthalmology, 55, 516-530. https://doi.org/10.1016/j.survophthal.2010.02.005

[28] Su, D. and Hubschman, J.P. (2017) A Review of Subthreshold Micropulse Laser and Recent Advances in Retinal Laser Technology. Ophthalmology \& Therapy, 6, 1-6. https://doi.org/10.1007/s40123-017-0077-7

[29] Flaxel, C., Bradle, J., Acott, T. and Samples, J.R. (2007) Retinal Pigment Epithelium Produces Matrix Metalloproteinases after Laser Treatment. Retina, 27, 629-634. https://doi.org/10.1097/01.iae.0000249561.02567.fd

[30] Brader, H.S. and Young, L.H. (2016) Subthreshold Diode Micropulse Laser: A Review. Seminars in Ophthalmology, 31, 30-39. https://doi.org/10.3109/08820538.2015.1114837

[31] Lavinsky, D., Cardillo, J.A., Jr., M.L., Dare, A., Farah, M.E. and Jr., B.R. (2011) Randomized Clinical Trial Evaluating mETDRS versus Normal or High-Density Micropulse Photocoagulation for Diabetic Macular Edema. Investigative Ophthalmology \& Visual Science, 52, 4314-4323. https://doi.org/10.1167/iovs.10-6828

[32] Luttrull, J.K. and Giorgio, D. (2012) Subthreshold Diode Micropulse Laser Photocoagulation (SDM) as Invisible Retinal Phototherapy for Diabetic Macular Edema: A Review. Current Diabetes Reviews, 8, 274-284. https://doi.org/10.2174/157339912800840523

[33] Frcs, S.S., Mrcophth, R.S., Mrcophth, A.T., Mrcophth, S.A. and Frcophth, M.H. (2007) Subthreshold Micropulse Diode Laser Photocoagulation for Clinically Significant Diabetic Macular Oedema: A Three-Year Follow Up. Clinical \& Experimental Ophthalmology, 35, 640-644.

https://doi.org/10.1111/j.1442-9071.2007.01566.x

[34] Brinkmann, R., Roider, J. and Birngruber, R. (2006) Selective Retina Therapy (SRT): A Review on Methods, Techniques, Preclinical and First Clinical Results. Bulletin De La Société Belge Dophtalmologie, 302, 51-69.

[35] Roider, J., Michaud, N.A., Flotte, T.J. and Birngruber, R. (1992) Response of the Retinal Pigment Epithelium to Selective Photocoagulation. Archives of Ophthalmolo$g y$, 110, 1786-1792. https://doi.org/10.1001/archopht.1992.01080240126045

[36] Sher, A., Jones, B.W., Huie, P., Paulus, Y.M., Lavinsky, D., Leung, L.S., et al. (2013) Restoration of Retinal Structure and Function after Selective Photocoagulation. Journal of Neuroscience, 33, 6800-6808. https://doi.org/10.1523/JNEUROSCI.1044-12.2013 
[37] Roider, J., Brinkmann, R., Wirbelauer, C., Laqua, H. and Birngruber, R. (2000) Subthreshold (Retinal Pigment Epithelium) Photocoagulation in Macular Diseases: A Pilot Study. British Journal of Ophthalmology, 84, 40-47. https://doi.org/10.1136/bjo.84.1.40

[38] Paulus, Y.M., Jain, A., Nomoto, H., Sramek, C., Gariano, R.F., Andersen, D., et al. (2011) Selective Retinal Therapy with Microsecond Exposures Using a Continuous Line Scanning Laser. Retina, 31, 380-388. https://doi.org/10.1097/IAE.0b013e3181e76da6

[39] Roider, J., Liew, S.H.M., Klatt, C., Elsner, H., Poerksen, E., Hillenkamp, J., et al. (2010) Selective Retina Therapy (SRT) for Clinically Significant Diabetic Macular Edema. Graefe's Archive for Clinical \& Experimental Ophthalmology, 248, 1263-1272. https://doi.org/10.1007/s00417-010-1356-3

[40] Elsner, H., Pörksen, E., Klatt, C., Bunse, A., Theisen-Kunde, D., Brinkmann, R., et al. (2006) Selective Retina Therapy in Patients with Central Serous Chorioretinopathy. Graefe's Archive for Clinical \& Experimental Ophthalmology, 244, 1638-1645. https://doi.org/10.1007/s00417-006-0368-5

[41] Nazari, H., Zhang, L., Zhu, D., Chader, G.J., Falabella, P., Stefanini, F., et al. (2015) Stem Cell Based Therapies for Age-Related Macular Degeneration: The Promises and the Challenges. Progress in Retinal \& Eye Research, 48, 1-39. https://doi.org/10.1016/j.preteyeres.2015.06.004

[42] Wood, J.P.M., Plunkett, M., Previn, V., Chidlow, G. and Casson, R.J. (2011) Nanosecond Pulse Lasers for Retinal Applications. Lasers in Surgery \& Medicine, 43, 499-510. https://doi.org/10.1002/lsm.21087

[43] Chidlow, G., Shibeeb, O., Plunkett, M., Casson, R.J. and Wood, J.P. (2013) Glial Cell and Inflammatory Responses to Retinal Laser Treatment: Comparison of a Conventional Photocoagulator and a Novel, 3-Nanosecond Pulse Laser. Investigative Ophthalmology \& Visual Science, 54, 2319-2332. https://doi.org/10.1167/iovs.12-11204

[44] Jobling, A.I., Guymer, R.H., Vessey, K.A., Greferath, U., Mills, S.A., Brassington, K.H., et al. (2015) Nanosecond Laser Therapy Reverses Pathologic and Molecular Changes in Age-Related Macular Degeneration without Retinal Damage. Faseb Journal Official Publication of the Federation of American Societies for Experimental Biology, 29, 696-710. https://doi.org/10.1096/fj.14-262444

[45] Guymer, R.H., Brassington, K.H., Dimitrov, P., Makeyeva, G., Plunkett, M., Xia, W., et al. (2014) Nanosecond-Laser Application in Intermediate AMD: 12-Month Results of Fundus Appearance and Macular Function. Clinical \& Experimental Ophthalmology, 42, 466-479. https://doi.org/10.1111/ceo.12247

[46] Casson, R.J., Raymond, G., Newland, H.S., Gilhotra, J.S. and Gray, T.L. (2012) Pilot Randomized Trial of a Nanopulse Retinal Laser versus Conventional Photocoagulation for the Treatment of Diabetic Macular Oedema. Clinical \& Experimental Ophthalmology, 40, 604-610. https://doi.org/10.1111/j.1442-9071.2012.02756.x

[47] Hu, Z., Zhang, H., Mordovanakis, A., Paulus, Y.M., Liu, Q., Wang, X., et al. (2017) High-Precision, Non-Invasive Anti-Microvascular Approach via Concurrent Ultrasound and Laser Irradiation. Scientific Reports, 7, Article No. 40243. https://doi.org/10.1038/srep40243

[48] Li, J. and Paulus, Y.M. (2018) Advances in Retinal Laser Therapy. International Journal of Ophthalmic Research, 4, 1-6. 